INPLASY

PROTOCOL

To cite: Song et al. Clinical efficacy of traditional Chinese medicine in advanced colorectal cancer: a systematic review and meta-analysis. Inplasy protocol 202140132. doi:

10.37766/inplasy2021.4.0132

Received: 25 April 2021

Published: 26 April 2021

Corresponding author:

Zhuo Song

drzhuosong2013@126.com

Author Affiliation:

Xiyuan Hospital of Chinese Academy of Chinese Medical Sciences

Support: China.

Review Stage at time of this submission: Preliminary searches.

Conflicts of interest: None declared.

\section{Clinical efficacy of traditional Chinese medicine in advanced colorectal cancer: a systematic review and meta-analysis}

Song, Z1; Zou, JH2; Tang, M33 Zhang, ZY4; Song, QY5; Yang, YF6; $\mathrm{Wu}, \mathrm{Y}^{7} ; \mathrm{Xu}, \mathrm{Y}^{8}$.

Review question / Objective: Effect of TCM loading intervention on clinical efficacy of advanced colorectal cancer compared with conventional treatment.

Condition being studied: The clinical efficacy of traditional Chinese medicine loading intervention on the treatment of advanced colorectal cancer, including oral or intravenous injection of traditional Chinese medicine preparation, advanced colorectal cancer patients with tumor, the control group was treated with conventional western medicine treatment, such as chemotherapy, supportive treatment, the intervention group was treated with traditional Chinese medicine on the basis of the control group, the research type was randomized controlled study, The study outcome indicators include at least one of the long-term efficacy, such as overall survival, progression free survival, 1-year survival, 3-year survival and 5-year survival. The analysis is not limited to the long-term efficacy, but the short-term efficacy, such as the objective remission rate and quality of life. The language of included literature is not limited.

INPLASY registration number: This protocol was registered with the International Platform of Registered Systematic Review and Meta-Analysis Protocols (INPLASY) on 26 April 2021 and was last updated on 26 April 2021 (registration number INPLASY202140132).

\section{INTRODUCTION}

Review question / Objective: Effect of TCM loading intervention on clinical efficacy of advanced colorectal cancer compared with conventional treatment.
Condition being studied: The clinical efficacy of traditional Chinese medicine loading intervention on the treatment of advanced colorectal cancer, including oral or intravenous injection of traditional Chinese medicine preparation, advanced colorectal cancer patients with tumor, the control group was treated with 
conventional western medicine treatment, such as chemotherapy, supportive treatment, the intervention group was treated with traditional Chinese medicine on the basis of the control group, the research type was randomized controlled study, The study outcome indicators include at least one of the long-term efficacy, such as overall survival, progression free survival, 1-year survival, 3year survival and 5-year survival. The analysis is not limited to the long-term efficacy, but the short-term efficacy, such as the objective remission rate and quality of life. The language of included literature is not limited.

\section{METHODS}

Search strategy: (clinical trial OR randomized controlled trial OR controlled clinical trial OR random allocation) AND (medicine, herbal OR plant extracts OR drugs, Chinese herbal OR plants, medicinal OR medicine, Chinese traditional) AND (colonic neoplasms OR rectal neoplasms OR colorectal neoplasms).

\section{Participant or population: Stage IV.}

Intervention: Traditional Chinese medicine including oral or intravenous injection preparation.

Comparator: Conventional treatment, such as chemotherapy, supportive treatment.

Study designs to be included: Randomized controlled trial.

Eligibility criteria: Non RCT; the treatment group also combined with other treatments such as acupuncture, massage, besides the control group; traditional Chinese medicine are external application; the outcome indicators did not meet the requirements, the data could not be statistically analyzed or the data were questionable.

Information sources: China National Knowledge Infrastructure, Wanfang Database, VIP Database, PubMed, Cochrane Library, Excerpta Medica
Database(EMBASE), and gray literatures such as conferences and dissertates.

Main outcome(s): Main outcome is longterm efficacy, such as overall survival, progression free survival, 1-year survival, 3year survival and 5-year survival.

Additional outcome(s): Objective remission rate, improvement of quality of life.

Data management: Endnote software.

Quality assessment / Risk of bias analysis: Risk bias assessment tools form Cochrane.

Strategy of data synthesis: RevMan5.4.1 form Cochrane.

Subgroup analysis: The subgroup analysis was divided into chemotherapy and no chemotherapy according to control group

Sensitivity analysis: Exclude one by one, to look for sources of heterogeneity.

Language: Language is not limited.

Country(ies) involved: China.

Keywords: Traditional Chinese medicine; advanced colorectal cancer; systematic review; clinical efficacy.

Dissemination plans: Publish an article.

Contributions of each author:

Author 1 - Zhuo Song.

Author 2 - Jianhua Zou.

Author 3 - Mo Tang.

Author 4 - Zhongyue Zhang.

Author 5 - Qingya Song.

Author 6 - Yufei Yang.

Author 7 - Yu Wu.

Author 8 - Yun Xu. 